\title{
Robotic Versus Open Ureteroneocystostomy: Is There a Robotic Benefit?
}

\author{
Peyton A. Skupin, BS, John T. Stoffel, MD, ${ }^{2}$ Bahaa S. Malaeb, MD, ${ }^{2}$ \\ Paholo Barboglio-Romo, MD, ${ }^{2}$ and Sapan N. Ambani, MD ${ }^{2}$
}

\begin{abstract}
Aim: We sought to compare the outcomes of patients who underwent an open $v s$ robotic ureteroneocystostomy for ureteral obstruction.

Methods: Retrospective review was performed on adult patients who underwent primary ureteroneocystostomy for obstruction from January 2012 to April 2018. Intraoperative outcomes of estimated blood loss (EBL) and operative time, as well as postoperative outcomes of catheter and stent duration, length of hospital stay, inpatient nurse-controlled opioid use, patient-controlled analgesia (PCA), and outpatient opioid prescription, complications, readmission, radiologic and clinical stricture recurrence, and follow-up, were compared. Among the open cohort, indications for an open approach were evaluated, identifying patients with prior complex open abdominal surgery that would make an open approach preferable.

Results: Open ureteroneocystostomy was performed in 27 patients compared with 18 who underwent a robotic approach. The open and robotic cohorts were not significantly different in age, gender, Charlson comorbidity index, stricture location or side, abdominal surgery (laparoscopic or open), pelvic radiation, or preoperative urinary tract infection. The robotic group had a significantly lower rate of prior open abdominal surgery. The robotic cohort had significantly lower EBL, length of stay (LOS), catheter duration, prescribed morphine milliequivalents (MME) at discharge, and rate of PCA usage. Among the open cohort, 13 (48\%) patients demonstrated indications making an open approach preferable. Comparing the robotic group with the remaining 14 open patients revealed a significantly lower rate of inpatient PCA use, prescribed MME at discharge, LOS, and catheter duration. Mean operative time was higher in the robotic group. EBL was not significantly different in this subanalysis.

Conclusions: Robotic ureteroneocystostomy provides similar outcomes when compared with an open approach in well-selected patients when assessing for recurrent ureteral obstruction or adverse events. Robotic surgery is associated with lower postoperative narcotic pain prescriptions at discharge, lower PCA usage, and shorter LOS, which are important benefits when compared with open surgery.
\end{abstract}

Keywords: ureteroneocystostomy, ureteral stricture, ureteral obstruction, ureteral reconstruction, robotics, laparoscopy approach

\section{Introduction}

$\mathbf{U}$ RETERAL INJURY AND stricture disease is an uncommon but morbid condition that often requires complex ureteral reconstruction as treatment. ${ }^{1}$ A definitive surgical option for repair of ureteral obstruction is ureteroneocystostomy, which historically was performed via an open approach with excellent success rates. ${ }^{2}$ With the availability of minimally invasive techniques, laparoscopic ureteroneocystostomy has been described as a feasible approach to managing ureteral reconstruction. ${ }^{3,4}$ Studies demonstrate that this approach is associated with a benefit in terms of hospitalization duration, intraoperative blood loss, and narcotic pain prescription while carrying no increased complications compared with the open approach. ${ }^{3,4}$

Robotic ureteroneocystostomy has gained popularity over a standard laparoscopic approach for ureteral surgery due to greater visibility, maneuverability, dexterity,

\footnotetext{
${ }^{1}$ University of Michigan Medical School, Ann Arbor, Michigan, USA.

${ }^{2}$ Department of Urology, University of Michigan, Ann Arbor, Michigan, USA.
} 
surgeon ergonomics, and ease of intracorporeal suturing. ${ }^{5-7}$ Few studies exist comparing the outcomes of robotic ureteroneocystostomy with an open approach. ${ }^{8} \mathrm{We}$ sought to compare the intraoperative and postoperative course of patients who underwent ureteroneocystostomy via robotic $v s$ an open approach. Multiple factors can influence a patient's candidacy for robotic surgery. We also sought to compare those open surgical patients without a history of complex open intraperitoneal surgery with robotic surgery patients. We hypothesized that patients who underwent robotic surgery would have equivalent outcomes with the potential for improved postoperative convalescence when compared with the open ureteroneocystostomy patients, and that these conclusions would persist when only comparing robotic patients with those open patients without a contraindication to a minimally invasive approach.

\section{Methods}

\section{Data and study population}

All patients who underwent a ureteroneocystostomy between January 2012 and March 2018 at a single institution were identified using billing codes. Of these 646 patients, 598 were excluded from the initial data set. Exclusion criteria were as follows: younger than 18 years, malignancy of the ureter or bladder, ureteroneocystostomy into a conduit or neobladder, renal transplant ureteroneocystostomy, history of complex urologic reconstruction (such as bladder augmentation cystoplasty), Boari flap, intravesical psoas hitch, bilateral ureteroneocystostomy, or a ureteroneocystostomy performed in conjunction with another major surgery. Three patients were converted from a robotic to open approach intraoperatively: two due to intra-abdominal adhesions, and one due to poor visualization and failure to progress; these three patients were also excluded from comparative analysis (Fig. 1).

\section{Outcomes}

After institutional review board (IRB) approval, we performed a retrospective chart review with interest in the following: patient demographics, Charlson comorbidity index, side, location, and etiology of obstruction, estimated blood loss (EBL) and operative time, length of hospital stay, inpatient nurse-controlled IV and oral opioid use, patient-controlled analgesia (PCA), and discharge opioid prescription, days with catheter after surgery, stent duration, complications, readmission rate, stricture recurrence demonstrated radiologically or need for further instrumentation, and time to follow-up. Among the open cohort, indications for an open approach were evaluated, identifying patients with prior complex open abdominal surgery that would make an open approach preferable.

\section{Statistical analysis}

Statistical analysis was performed using SPSS $^{\circledR}$ statistical software (IBM, Armonk, NY). Open and robotic cohorts were compared using independent samples parametric $t$ test, Mann-Whitney U test, and chi-squared test when appropriate, with $p$-value $<0.05$ considered statistically significant.

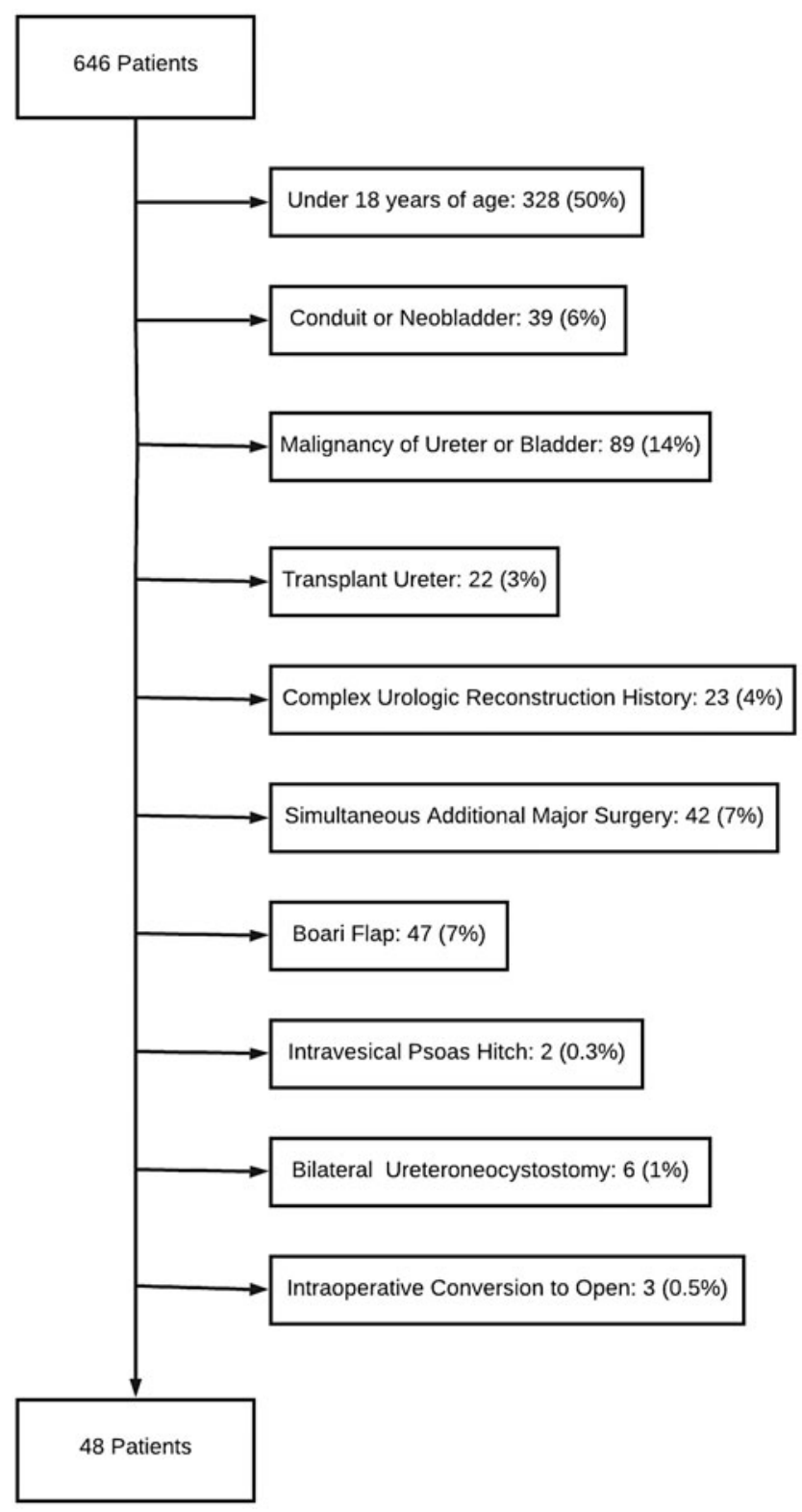

FIG. 1. Exclusion criteria. Six hundred forty-six patients were identified using CPT codes 50780, 50782, 50783, $50785,50947,50948$, and 50949. CPT $=$ current procedural terminology.

\section{Results}

Forty-five patients met the inclusion criteria. Twentyseven underwent an open ureteroneocystostomy, and 18 underwent robotic reconstruction. The decision to proceed with either robotic or open surgery was made by the primary surgeon. The 27 open patients were performed by 6 urologists, while the 18 robotic patients were performed by 5 urologists. Of the open cohort, $23(85.2 \%)$ of ureteroneocystostomies were performed via midline incision, and 4 $(14.8 \%)$ were performed via Pfannenstiel incision. Of note, the open surgery patients had a significantly higher rate of prior open abdominal surgery, $13(48.1 \%)$ as opposed to 2 $(11.1 \%)$ in the robotic group. Demographic data are found in Table 1. 
Table 1. Patient Demographics

\begin{tabular}{|c|c|c|c|}
\hline & $\begin{array}{c}\text { Open } \\
(\mathrm{n}=27)\end{array}$ & $\begin{array}{l}\text { Robotic } \\
(\mathrm{n}=18)\end{array}$ & $\mathrm{p}$ \\
\hline Age (years), mean (SD) & $48.3(14.5)$ & $53.1(15.2)$ & 0.29 \\
\hline Gender, $n(\%)$ & & & 0.46 \\
\hline Male & $5(18.5)$ & $5(27.8)$ & \\
\hline Female & $22(81.5)$ & $13(72.2)$ & \\
\hline $\begin{array}{l}\text { Charlson comorbidity } \\
\text { index, } n(\%)\end{array}$ & & & 0.75 \\
\hline 0 & $9(33.3)$ & $7(38.9)$ & \\
\hline 1 to 3 & $12(44.4)$ & $6(33.3)$ & \\
\hline$\geq 4$ & $6(22.2)$ & $5(27.8)$ & \\
\hline Stricture side, $n(\%)$ & & & 0.11 \\
\hline Left & $14(51.9)$ & $5(27.8)$ & \\
\hline Right & $13(48.1)$ & $13(72.2)$ & \\
\hline $\begin{array}{l}\text { Stricture location, } \\
n(\%)\end{array}$ & & & 0.13 \\
\hline Proximal & $0(0)$ & $0(0)$ & \\
\hline Middle/iliac & $6(22.2)$ & $1(5.6)$ & \\
\hline Distal & $21(74.1)$ & $17(94.4)$ & \\
\hline $\begin{array}{l}\text { Prior abdominal surgery, } \\
\quad n(\%)\end{array}$ & $23(85.2)$ & $13(72.2)$ & 0.29 \\
\hline $\begin{array}{l}\text { Prior open abdominal } \\
\quad \text { surgery, } n(\%)\end{array}$ & $13(48.1)$ & $2(11.1)$ & 0.01 \\
\hline $\begin{array}{l}\text { Prior pelvic radiation, } \\
n(\%)\end{array}$ & $1(3.7)$ & $1(5.6)$ & 0.77 \\
\hline $\begin{array}{l}\text { Preoperative UTI, } \\
n(\%)\end{array}$ & $8(29.6)$ & 7 (38.9) & 0.57 \\
\hline
\end{tabular}

$\mathrm{SD}=$ standard deviation; $\mathrm{UTI}=$ urinary tract infection.
In the robotic cohort, there was a lower EBL after the operation, with a median of $50 \mathrm{cc}$ compared with $100 \mathrm{cc}$ in the open cohort $(p=0.006)$. No patients required a blood transfusion. Patients who underwent a robotic ureteroneocystostomy also had a shorter admission postoperatively, with the median length of stay (LOS) at 1.5 days, as opposed to 3 days in the open group $(p<0.001)$. Robotic cohort patients also had a urethral catheter after surgery for a significantly shorter time, with a median of 1 day in the robotic group and 9 days in the open group $(p=0.001)$. There were no significant differences in complication rates as an inpatient or 30 or 90 days postoperatively. No differences in readmission rate or stricture recurrence were detected (Table 2).

Narcotic pain usage of patients as an inpatient as well as prescription narcotics given at discharge was compared.

.13 Patients who underwent a robotic ureteroneocystostomy had a significantly lower rate of PCA usage after their operation $(5.6 \%$ vs $55.6 \%, p<0.001)$. In addition, patients in the robotic cohort were prescribed an average of 336.4 morphine milliequivalents (MME) of narcotics at discharge, significantly lower than the open cohort (535.6 MME, $p=0.032$ ) (Table 2). The groups did not differ significantly in inpatient nurse-controlled IV and oral narcotic administration $(p=0.18)$.

Among the open cohort, $14(52 \%)$ patients did not have a history of complex intraperitoneal abdominopelvic surgery or absolute contraindication to a minimally invasive approach. It was presumed that these patients underwent an open approach based on the preferences of the patient or surgeon. We compared these 14 patients with the robotic surgery cohort. Analysis revealed a significantly lower rate of

TABLE 2. Results

\begin{tabular}{|c|c|c|c|}
\hline & Open $(\mathrm{n}=27)$ & Robotic $(\mathrm{n}=18)$ & $\mathrm{p}$ \\
\hline Operative time (minutes), mean (SD) & $209.6(128.6)$ & $195.0(49.5)$ & 0.65 \\
\hline Inpatient nurse-controlled morphine milliequivalents, mean (SD) & $367.0(304.4)$ & $263.4(120.5)$ & 0.18 \\
\hline Inpatient use of patient-controlled analgesia, $n(\%)$ & $15(55.6)$ & $1(5.6)$ & $<0.001$ \\
\hline Prescribed morphine milliequivalents at discharge, mean (SD) & $535.6(352.7)$ & $336.4(172.3)$ & 0.03 \\
\hline Estimated blood loss (cc), median (range) & $100(25-1300)$ & $50(10-150)$ & 0.006 \\
\hline Length of stay (days), median (range) & $3(1-11)$ & $1.5(1-4)$ & $<0.001$ \\
\hline Catheter duration (days), median (range) & $9(1-26)$ & $1(1-19)$ & 0.001 \\
\hline Stent duration (days), median (range) & $33(22-119)$ & $31.5(18-53)$ & 0.59 \\
\hline Clavien-Dindo classification inpatient, $n(\%)$ & & & 0.34 \\
\hline No complication & 23 & 17 & \\
\hline Grade I to II & 4 & 1 & \\
\hline Grade III to IV & 0 & 0 & \\
\hline Clavien-Dindo classification 30 days, $n(\%)$ & & & 0.44 \\
\hline No complication & 19 & 12 & \\
\hline Grade I to II & 6 & 6 & \\
\hline Grade III to IV & 1 & 0 & \\
\hline Clavien-Dindo classification 90 days, $n(\%)$ & & & 0.90 \\
\hline No complication & 17 & 11 & \\
\hline Grade I to II & 7 & 6 & \\
\hline Grade III to IV & 2 & 1 & \\
\hline 90-Day readmission, $n(\%)$ & $7(26.9)$ & $5(27.8)$ & 0.71 \\
\hline Stricture recurrence, $n(\%)$ & $1(3.7)$ & $0(0.0)$ & 0.50 \\
\hline Radiologic follow-up (months), mean (SD) & $12.1(13.3)$ & $10.6(12.1)$ & 0.70 \\
\hline Symptomatic follow-up (months), mean (SD) & $19.9(17.8)$ & $14.1(14.9)$ & 0.26 \\
\hline
\end{tabular}


inpatient PCA use $(5.5 \%$ vs $35.7 \%, p=0.030)$, prescribed MME at discharge (336.39 vs 555.71, $p=0.046)$, LOS (1.5 vs 2, $p=0.004)$, and urethral catheter duration in days after surgery (1 vs 9.5, $p=0.002$ ) in the robotic group. Operative time (minutes) was significantly longer in the robotic group (194.9 vs $157.4 p=0.022)$. EBL (cc) was not significantly different in this analysis (50 vs 50, $p=0.319$ ). There were no significant differences in complication rates as an inpatient, or 30 or 90 days postoperatively. No differences in readmission rate or stricture recurrence were detected.

\section{Discussion}

Robotic ureteroneocystostomy is gaining popularity over a standard laparoscopic or open approach. In this study, we compared patients who underwent open ureteroneocystostomy $v s$ those who had a robotic approach and found no detectable difference in postoperative complications or recurrent obstruction. The robotic group had a shorter LOS, shorter urethral catheter duration, and lower narcotic pain prescriptions. When patients who had prior complex open intraperitoneal surgery making an open approach preferable were excluded from analysis, these findings persisted. Robotic ureteroneocystostomy is a safe, viable procedure with comparable outcomes with an open approach.

In our study, patients with ureteral stricture disease who underwent a robotic ureteroneocystostomy had similar complication rates and there were no differences in readmission rate or stricture recurrence. Potential benefits of the robotic approach included shorter LOS, lower inpatient PCA use, and decreased opioid prescriptions at discharge. A shorter urethral catheter duration was also noted; however, it is important to state that this could reflect a difference in practice pattern rather than solely an advantage of a robotic approach. A decreased LOS and improved convalescence in the robotic group likely enable the patient to tolerate an earlier removal of the urethral catheter, but the difference in catheter duration likely does not reflect any difference in the quality of the ureteral anastomosis or bladder function that would require a prolonged urethral catheter in the open cohort.

Our findings support the current literature on robotic surgery for urologic procedures, including ureteroneocystostomy. Fifer and colleagues ${ }^{9}$ published a series of 55 patients who underwent robotic ureteral reconstructive procedures distal to the ureteropelvic junction. All cases were effectively completed robotically with no open conversions and no intraoperative complications. Median blood loss was $50 \mathrm{~mL}$. The average hospital stay was 1.6 days, with $71 \%$ discharged on postoperative day 1 . There were two serious complications and three failures. Hemal and coworkers ${ }^{6}$ describe 44 robotic procedures of diverse ureteral pathologies, including ureteroneocystostomy. Mean blood loss was $98.2 \mathrm{~mL}$ (range: $<50-400$ ). There were no urine leaks (range: $1-2.5$ ) and mean hospital stay was 2.4 days (range: $1-6$ ). Complications included one case of sepsis and one antibiotic-induced infection. Operative success as defined by symptom resolution and imaging was $100 \%$. These studies, while not a comparative analysis, support the feasibility of robotic ureteral reconstruction. We found similar efficacy in the robotic approach in our study.
Comparative analyses between robotic and open approaches are subject to selection bias, and our study is no different. In an attempt to evaluate cohorts that are more comparable, we identified patients who lacked a strong contraindication to a robotic approach, in this case prior complex open intraperitoneal surgery, but were still performed open based on patient and physician preference. When compared with the robotic cohort, the differences in LOS, catheter duration, PCA usage, and MME at discharge persisted, supporting the notion that the approach plays a role in varied postoperative outcomes, and cannot be solely explained by open ureteroneocystostomy patients having a more complex abdomen.

Our study does have several limitations. Our cohort size is small, and larger studies are required to validate our findings. This study was retrospective and therefore is subject to several biases. Aside from stricture location, other factors that contribute to stricture complexity could not be measured and therefore may confound our results. Although a prospective randomized trial would provide definitive results, this would be challenging to perform as there are several factors that influence the decision to perform this surgery via an open or robotic approach. Three patients required conversion from robotic to open, which reveals that a patient's candidacy for a robotic ureteroneocystostomy is a complex decision that cannot always be accounted for by surgical history or radiologic imaging. Related to this, changing practice patterns at our institution may have played a role in the surgeon's decision to operate robotically, as ureteroneocystostomy was not regularly performed robotically earlier in the cohort. It is prudent to address the fact that the differences in postoperative outcomes we observed may be due to practice patterns at our institution, particularly surrounding catheter duration. However, there was no difference in complication rates or stricture recurrence, supporting the safety of a robotic approach and its associated practice patterns.

\section{Conclusion}

Robotic surgery for ureteroneocystostomy is a safe, comparable alternative to open surgery with similar complication and failure rates and potential benefits in postoperative convalescence.

\section{Author Disclosure Statement}

No competing financial interests exist.

\section{Funding Information}

No funding was received for this article.

\section{References}

1. Elliott SP, McAninch JW. Ureteral injuries: External and iatrogenic. Urol Clin North Am 2006;33:55-66.

2. Stühler V, Bedke J, Stenzl A. Surgical reconstruction of the ureter. Urologe A 2019;58:651-657.

3. Han CM, Tan HH, Kay N, et al. Outcome of laparoscopic repair of ureteral injury: Follow-up of twelve cases. J Minim Invasive Gynecol 2012;19:68-75.

4. Rassweiler JJ, Gözen AS, Erdogru T, et al. Ureteral ureteroneocystostomyation for management of ureteral strictures: A retrospective comparison of laparoscopic and open techniques. Eur Urol 2007;51:512-523. 
5. Windsperger AP, Duchene DA. Robotic reconstruction of lower ureteral strictures. Urol Clin North Am 2013;40: 363-370.

6. Hemal AK, Nayyar R, Gupta NP, et al. Experience with robot assisted laparoscopic surgery for upper and lower benign and malignant ureteral pathologies. Urology 2010;76: 1387-1393.

7. Gellhaus PT, Bhandari A, Monn MF, et al. Robotic management of genitourinary injuries from obstetric and gynaecological operations: A multi-institutional report of outcomes. BJU Int 2015;115:430-436.

8. Phillips EA, Wang DS. Current status of robot-assisted laparoscopic ureteral ureteroneocystostomyation and reconstruction. Curr Urol Rep 2012;13:190-194.

9. Fifer GL, Raynor MC, Selph P, et al. Robotic ureteral reconstruction distal to the ureteropelvic junction: A large single institution clinical series with short-term follow up. J Endourol 2014;28:1424-1428.
Address correspondence to: Sapan N. Ambani, MD Department of Urology University of Michigan 1500 E. Medical Center Drive, SPC 5330 Ann Arbor, MI 48109

USA

E-mail: sapan@med.umich.edu

$\begin{aligned} & \text { Abbreviations Used } \\ \mathrm{EBL} & =\text { estimated blood loss } \\ \mathrm{LOS} & =\text { length of stay } \\ \mathrm{MME} & =\text { morphine milliequivalents } \\ \mathrm{PCA} & =\text { patient-controlled analgesia }\end{aligned}$

